\title{
ỨNG DỤNG IOT TRONG HỆ THỐNG QUẢN LÝ VÀ GIÁM SÁT NGÔI NHÀ THÔNG MINH THEO THỜI GIAN THỤ̉C
}

\author{
TRẦ THỊ MINH KHOA, NGUYỄN CAO ANH MINH, NGUYẼ̃N TH!̣ HẬU \\ Khoa Công nghệ thông tin, Truờng Đại học Công nghiệp thành phố Hồ Chí Minh \\ ttmkhoa@iuh.edu.vn
}

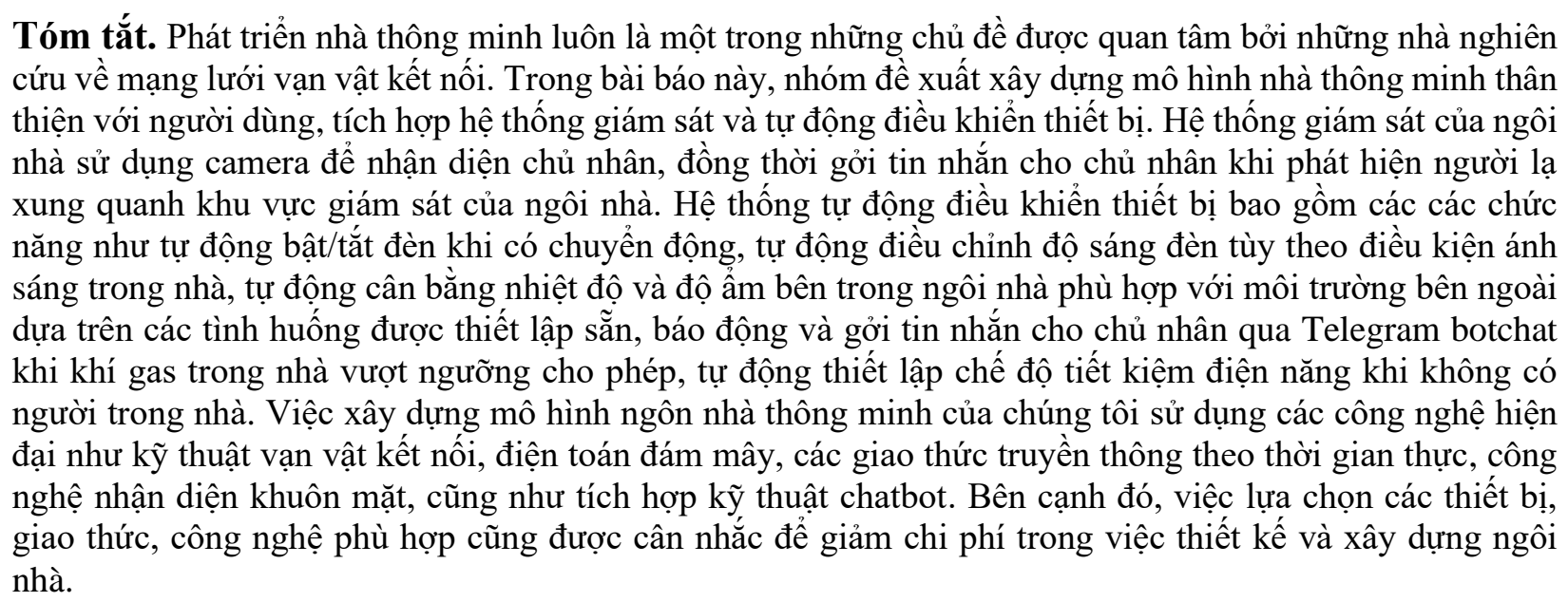

Từ khóa. Mạng lưới vạn vật kết nối, nhà thông minh, điện toán đám mây, giao thức truyền thông thời gian thực, chatbot.

\section{INTERNET OF THINGS ENABLES REAL TIME SMART HOME MONITORING SYSTEM}

\begin{abstract}
Smart home development is one of the hot topics that interested to IoT researchers. In this paper, we propose a human friendly-smart home integrated with security monitoring system as well as automatic devices controlling system. The security monitoring system use smart camera to identify stranger people and immediately send alert message to the owner Telegram botchat with the image and its information. The automatic devices controlling system with functions for devices remote control through the web or voice control. The functions include turn the light on/off, adjust the light on/off when human there is human movement, manual or automatically adjust the light level according to the brightness of the room, automatically balancing the temperature and humidity inside the house with the outside environment based on the user's built-in scenario, siren warning and sending alarm message via Telegram botchat when indoor gas exceeds the safe threshold. Our friendly-smart home is developed using current technologies such as internet of things, cloud computing and real time communication protocols, facial recognition technology, chatbot integrated technique.
\end{abstract}

Keywords. Internet of thing, smart house system, cloud technique, security monitoring syste

\section{GIỚI THIỆU}

Internet vạn vật (Internet of Things - IoT) là hệ thống các thiết bị máy tính kết nối với bất kỳ đối tượng hay vật thể nào. "Vạn vật" có thể bao gồm các máy móc, vật thể, động vật,... hoặc những thứ có liên quan đến nhau, được cung cấp một định danh duy nhất trong hệ thống và có khả năng truyền dữ liệu qua mạng mà không cần sự tương tác giữa con người với con người hay giữa con người với máy tính $[1,2]$. Nhà thông minh (Smart Home) bao gồm các hệ thống con nhằm phục vụ cuộc sống của con người thông qua các công nghệ hiện đại. Mục tiêu chính của hệ thống nhà thông minh là việc tích hợp các hệ thống, dịch vụ, và công 
cụ quản lý cung cấp cho con người một ngôi-nhà-sống thoải mái, tiện lợi và an toàn $[3,4]$. Khi công nghệ ngày càng phát triển xuất hiện nhiều hướng tiếp cận trong việc xây dựng mô hình nhà thông minh. Nhóm tác giả của bài báo [3] trình bày tổng quan các nghiên cứu về ứng dụng internet vạn vật trong việc thiết kế kiến trúc và triển khai các hệ thống nhà thông minh. Từ các hệ thống nhà thông minh cổ điển ở mức tự động, bán tự động,... đến các hệ thống nhà thông minh tự vận hành, xử lý tập trung dựa trên bộ các quy luật được thiết lập. Kiến trúc hệ thống cũng ngày càng phức tạp hơn nhằm đảm bảo tính hiệu quả, thuận tiện cũng như tính bảo mật của hệ thống. Bài báo [4] cho rằng nhà thông minh được phát triển dựa trên nền tảng vạn vật kết nối, các kỹ thuật máy tính, kỹ thuật điều khiển, kỹ thuật hiển thị hình ảnh, và kỹ thuật giao tiếp để kết nối các thiết bị vào mạng nhằm đáp ứng nhu cầu tự động hoá và cung cấp sự thuận lợi hơn cho quá trình quản lý và điều khiển.

Phát triển hệ thống nhà thông minh có khả năng tự động điều khiển theo điều kiện môi trường hoặc theo bất kỳ nhu cầu của người dùng là một trong những vấn đề được quan tâm của các nhà nghiên cứu, các kỹ sư nói riêng và các doanh nghiệp, người sử dụng nói chung. Việc áp dụng các kỹ thuật công nghệ thông tin tiên tiến vào quá trình phát triển và triển khai hệ thống nhà thông minh hiệu quả, hợp lý cũng là một thách thức lớn. Trong bài báo này, nhóm tác giả tìm hiểu, đề xuất và triển khai một hệ thông nhà thông minh tự động quy mô nhỏ với các chức năng thông dụng như: 1-nhận diện khuôn mặt chủ nhà, cảnh báo khi có người lạ xuất hiện trước cửa nhà, 2-điều khiên các thiết bị trong nhà bằng giọng nói, 3-tự động cân bằng nhiệt độ bên trong ngôi nhà phù hợp với nhiệt độ bên ngoài, 4-tự động thiết lập chế độ tiết kiệm điện năng khi không có người trong nhà, 5 -cung cấp ứng dụng web hô̂ trợ người dùng quản lý và điều khiển các thiêt bị trong nhà từ xa qua mạng.

\section{THIẾT KẾ VÀ XÂY DỬNG MÔ HİNH NHÀ THÔNG MINH HIỆN ĐẠI}

\subsection{Giới thiệu}

Hiện nay, ở Việt Nam, giải pháp xây dựng nhà thông minh với hệ thống điều khiển và giám sát thông qua internet đang phát triển vượt trội và nhận được sự quan tâm của các nhà nghiên cứu, nhà đầu tư, người dùng cũng như sinh viên các ngành công nghệ thông tin. Tuỳ vào kỹ thuật sử dụng, khả năng tài chính và các yêu cầu cần thiết mà nhiều hệ thống nhà thông minh khác nhau được thiết kế và xây dựng.

Để ứng dụng kỹ thuật IoT triển khai và phát triển ngôi nhà thông minh tích hợp hệ thống giám sát an ninh và điều khiển thiết bị tự động, nhóm sử dụng các công nghệ như lập trình thiết bị Arduino, kỹ thuật điện toán đám mây, kỹ thuật nhận diện khuôn mặt, nhận dạng giọng nói, kỹ thuật tích hợp chatbot vào hệ thống, các kỹ thuật xây dựng web để điều khiển hệ thống thông qua mạng internet.

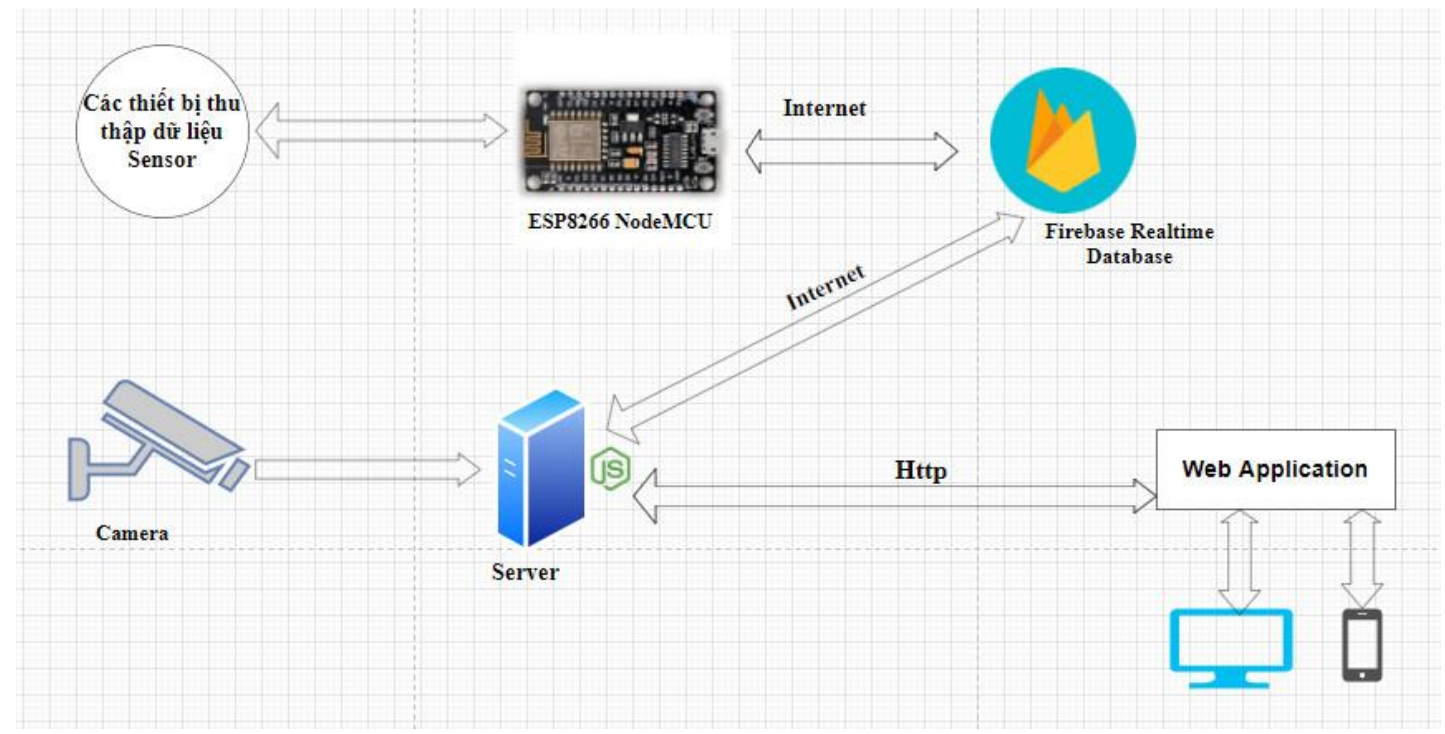

Hình 1. Mô hình kiến trúc IoT của hệ thống

Kiến trúc IoT tổng quát của mô hình được thể hiện qua Hình 1, bao gồm 5 khối:

- Khối cảm biến: Gồm các thiết bị có chức năng cảm biến và thu thập thông tin. 
- Khối điều khiển: Gồm thiết bị điều khiển - cổng vào của dữ liệu, đưa dữ liệu kết nối với nơi khối xử lý và lưu trữ. Nhóm sử dụng ESP8266 NodeMCU Lua CP2102

- Khối truyền dữ liệu: Bao gồm các giao thức kết nối giữa thiết bị điều khiển, thiết bị lưu trữ và xử lý dữ liệu. Trong Hình 1 , khối này là các giao thức truyền dữ liệu từ thiết bị điều khiển lên cơ sở dữ liệu lưu trữ Firebase Realtime Database, máy chủ Server, hay các lệnh điều khiển từ ứng dụng người dùng tới các thiết bị.

- Khối lưu trữ nơi lưu trữ và xử lý dữ liệu theo thời gian thực: Trong hệ thống, nhóm sử dụng cơ sở dữ liệu Firebase Realtime Database.

- Khối ứng dụng: với trang web hỗ trợ điều khiển các thiết bị từ xa hoặc ứng dụng điện thoài để điều khiển thiết bị qua mạng internet.

Với kiến trúc này sẽ quản lý, điều khiển các chức năng của một ngôi nhà, bao gồm: điều khiển bật/tắt đèn khi có chuyển động, điều khiển máy lạnh bằng giọng nói, cân bằng nhiệt độ bên trong ngôi nhà với điều kiện môi trường bên ngoài, thông báo nhiệt độ và độ ẩm, dự báo thời tiết bên ngoài, cảnh báo khí gas vượt ngưỡng và gởi tin nhắn cảnh báo cho người dùng. Hệ thống camera giám sát an ninh sử dụng kỹ thuật nhận diện khuôn mặt và gởi hình ảnh người lạ xuất hiện trong khu vực giám sát của ngôi nhà cho người dùng qua Telegram chatbot.

\subsection{Yêu cầu và thiết kế phần cứng}

Để thiết kế hệ thống sẽ gồm các thiết bị phần cứng được liệt kê trong Bảng 1.

Bảng 1. Yêu cầu phần cúng

\begin{tabular}{|l|l|}
\hline \multicolumn{1}{|c|}{ Thiết bị } & \multicolumn{1}{c|}{ Chức năng } \\
\hline ESP8266 NodeMCU tích hợp WiFi 2.4 GHz & $\begin{array}{l}\text { Kết nối và điều khiển các thiết bị cảm biến, thông qua } \\
\text { WiFi }\end{array}$ \\
\hline Đèn Led & Hiển thị ánh sáng đèn bật tắt \\
\hline Cảm biến nhiệt độ, độ ẩm DHT22 & Đo nhiệt độ, độ ẩm trong căn nhà \\
\hline Cảm biến khí gas MQ2 & Phát hiện rò rỉ khí Gas \\
\hline Camera & Quan sát, ghi dữ liệu hình ảnh \\
\hline $\begin{array}{l}\text { Cảm biến chuyển động hồng ngoại PIR } \\
\text { Sensor HC-SR501 }\end{array}$ & Xác định chuyển động của con người trong khu vực \\
\hline Led hồng ngoại & Thu và phát bức xạ hồng ngoại \\
\hline Cảm biến ánh sáng Photodiod Light Sensor & Phát hiện ánh sáng trong môi trường và cường độ sáng. \\
\hline
\end{tabular}

Sơ đồ kết nối các thiết bị được mô tả như trong các Hình 2, Hình 3, Hình 4, Hình 5 .

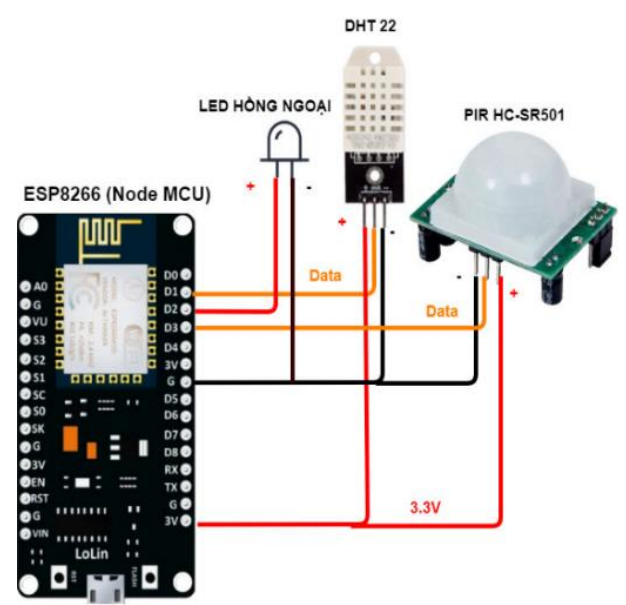

Hình 2. So đồ kết nối chân giữa cảm biến PIR, DHT22, led hồng ngoại và ESP8266. 
Hình 2 - Sơ đồ kết nối chân giữa cảm biến PIR, DHT22, led hồng ngoại và ESP8266. xây dựng chức năng giám sát nhiệt độ, độ ẩm trong ngôi nhà và điều khiến máy lạnh theo hai chế độ. Cảm biến DHT22 đo nhiệt độ, độ ẩm trong nhà đồng thời cung cấp dữ liệu độ ẩm xây dựng chế độ điều khiển tự động máy lạnh theo độ ẩm trong nhà đã thu thập qua cảm biến DHT22, với chế độ tự động điều khiển ở máy lạnh thêm điều kiện có chuyển động của con người mới thực hiện mở máy lạnh, dữ liệu theo dõi chuyển động người thu thập thông qua cảm biến chuyển động hồng ngoại HC-SR501 và cập nhật trạng thái máy lạnh thông qua led hồng ngoại. Led hồng ngoại thu thập tín hiệu của máy lạnh để xử lý và phát ra tín hiệu điều khiển máy lạnh.

Hình 3 - Sơ đồ kết nối chân giữa cảm biến MQ2, còi và ESP8266. Cảm biến khí gas MQ2 thu thập dữ liệu nồng độ khí gas đơn vị đo ppm, thu thập và gửi dữ liệu đến ESP8266 NodeMCU xử lý dữ liệu.

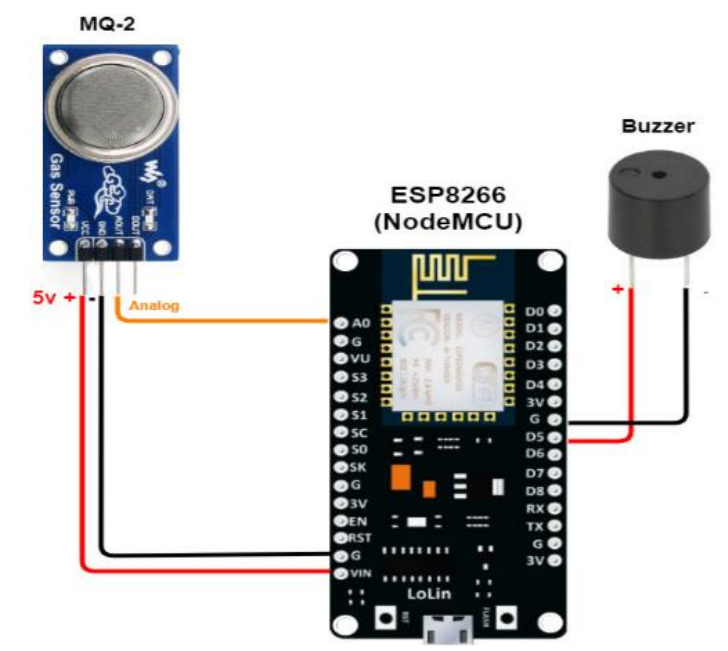

Hình 3. So đồ kết nối chân giữa cảm biến MQ2, còi và ESP8266

Hình 4 - Sơ đồ kết nối chân giữa cảm biến ánh sáng, led và ESP8266. Chức năng điều khiển đèn với các mức độ sáng phù hợp sinh hoạt, thu thập dữ liệu mức độ ánh sáng trong khu vực thông qua cảm biến ánh sáng Photodiod và gửi đến ESP8266 NodeMCU để xử lý cường độ sáng xung quanh và xây dựng kịch bản hiển thị mức độ sáng phù hợp thông qua đèn led với cường độ sáng khác nhau.

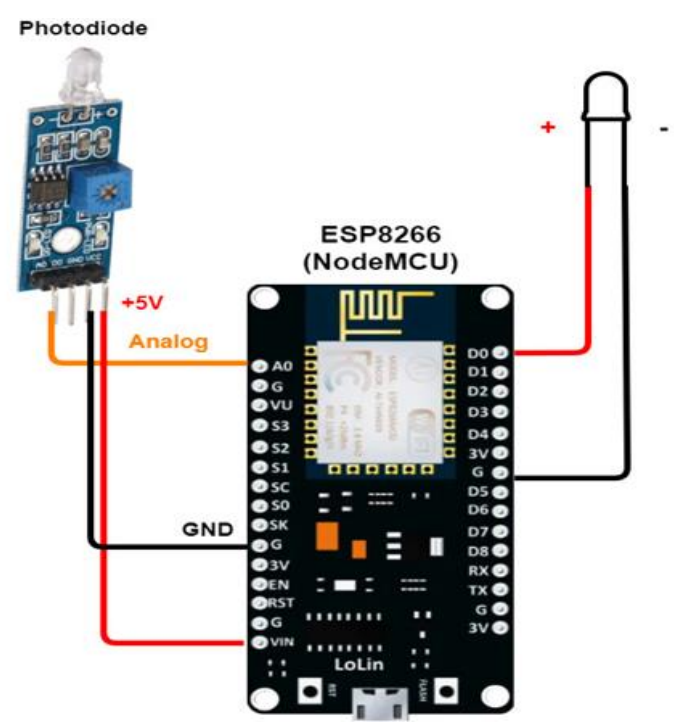

Hình 4. So đồ kết nối chân giũua cảm biến ánh sáng, led và ESP8266.

Hình 5 - Sơ đồ kết nối chân giữa cảm biến chuyển động, led với ESP8266. Điều khiển đèn thông qua chuyển động của người, thu thập dữ liệu con người chuyển động thông qua cảm biến PIR HC-SR501 gửi đến 
ESP8266 NodeMCU để xử lý và hiển thị bật, tắt đèn led theo chuyển động của con người, nếu có chuyển động người bật đèn và không có chuyển động của người thì tắt đèn.

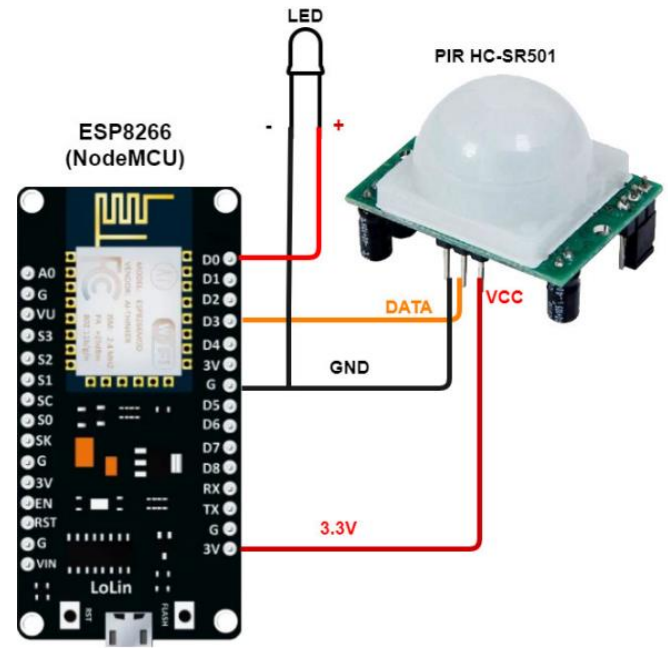

\subsection{Thiết kế phần mềm}

Hình 5. So đồ kết nối chân giữa cảm biến chuyển động, led với ESP8266.

Quá trình thực hiện xây dựng chức năng nhóm ứng dựng các ứng dụng công nghệ kỹ thuật dễ tiếp cận như Arduino IDE kết nối các cảm biến, xử lý dữ liệu thô. Firebase Realtime Database nơi lưu trữ dữ liệu các thông số về nhiệt độ, độ ẩm, khí gas đã được xử lý và dùng Firebase Realtime Database hỗ trợ về Hosting và cung cấp CDN xây dựng trang web để điều khiển các thiết bị từ xa và giám sát ngôi nhà thông qua camera.

Arduino IDE là một phần mềm giúp chúng ta nạp code đã viết vào board mạch và thực thi ứng dụng. Arduino IDE là chữ viết của Arduino Integrated Development Environment - một công cụ lập trình với các board mạch Arduino. Nó bao gồm các phần chính là: Editor (trình soạn thảo văn bản, dung để viết code), Debugger (công cụ giúp tìm kiếm và sửa lỗi phát sinh khi build chương trình), Compiler hoặc Interpreter (công cụ giúp biên dịch code thành ngôn ngữ mà vi điều khiển có thể hiểu được và thực thi code theo yêu cầu người dùng) [5].

Firebase Realtime Database lưu trữ thông tin từ các cảm biến và là trung gian để gởi dữ liệu theo truy vấn của ứng dụng web và ứng dụng di động. Firebase Realtime Database là một cơ sở dữ liệu NoSQL trên đám mây cũng cho phép đồng bộ hóa dữ liệu giữa các user trong thời gian thực. Nhận dữ liệu JSON và đồng bộ thời gian thực của tất cả các kết nối client. Realtime Database cung cấp SDKs cho cả web và mobile cho phép xây dựng ứng dụng mà không cần Server [6].

Nguyên lý hoạt động của các chức năng của hệ thống điều khiển các thiết bị trong ngôi nhà được mô tả như sau:

- Nguyên lý hoạt động của chức năng điều khiển máy lạnh, Hình 6 , người dùng điều khiển từ xa các chức năng của điều khiển máy lạnh được hiển thị trên web, dữ liệu điều khiển chức năng của máy lạnh được lưu lại tại Firebase Realtime Database, tại đây dữ liệu được truyền về ESP8266 đọc tín hiệu và chuyển đổi tín hiệu gửi cho cảm biến hồng ngoại, led phát hồng ngoại phát tín hiệu điều khiển máy lạnh, chuyển đổi trạng thái của máy lạnh theo tín hiệu điều khiển.

- Nguyên lý hoạt động của chức năng giám sát nhiệt độ, Hình 7 , nhiệt độ và độ ẩm trong ngôi được cảm biến nhiệt độ DHT22 đo, truyền tín hiệu qua ESP8266 NodeMCU. Tại đây, thông qua kết nối WiFi truyền dữ liệu nhiệt độ, độ ẩm lên Firebase Realtime Database để lưu trữ. Dữ liệu thông qua Server xử lý và hiển thị nhiệt độ, độ ẩm trên giao diện web. Dữ liệu được hiển thị theo hai dạng đó là dữ liệu thể hiện bằng biểu đồ dạng đường hiển thị theo thời gian thực đo được - thông tin nhiệt độ, độ ẩm cao nhất và thấp nhất trong ngày.

- Nguyên lý hoạt động của chức năng điều khiển đèn, Hình 8 , thông qua giao diện điều khiển đèn tắt, mở trên trang web, khi nhận lệnh điều khiển, truyền qua nơi lưu trữ Firebase nhận dữ liệu thay đổi gửi về cho ESP8266 đọc lệnh và cập nhật trạng thái của đèn. Hệ thống điều khiển đèn thông qua cảm biến chuyển động hồng ngoại PIR HC-SR501 nhận biết chuyển động thông qua thân nhiệt người để điều khiển tắt mở đèn trong nhà vệ sinh. 


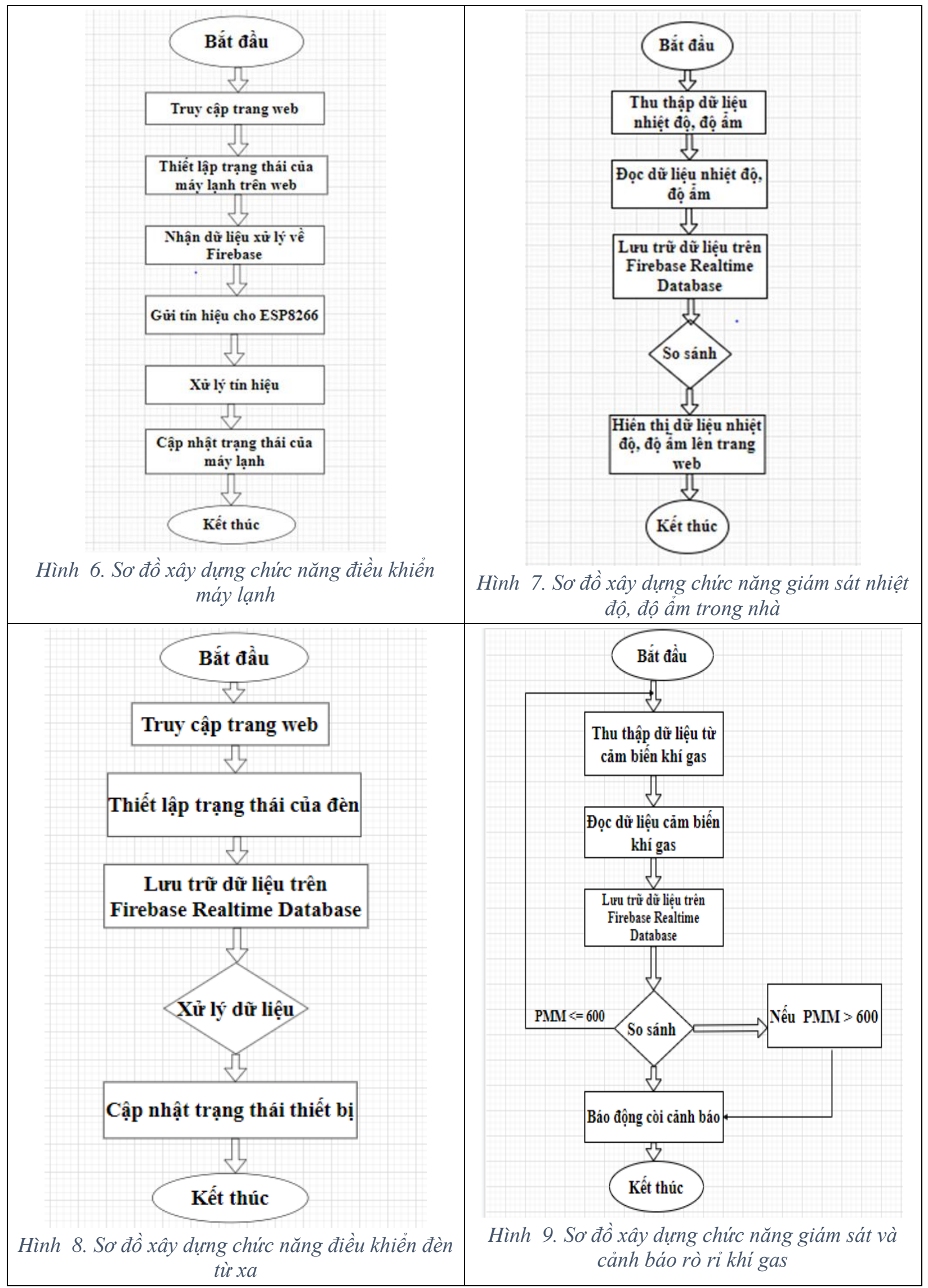


- Nguyên lý hoạt động của chức năng giám sát gas trong nhà, Hình 9, nồng độ khí gas trong ngôi nhà được đo bởi thiết bị cảm biến MQ2, nồng độ gas được đo tính theo PPM thể hiện trên trang web cho người dùng dễ theo dõi, giám sát.

- Nguyên lý hoạt động của hệ thống camera an ninh giám sát, Hình 10, camera được kết nối với server ghi nhận hình ảnh trong bán kính khu vực cho phép, dữ liệu hình ảnh được ghi lại gửi đến server để phân tích các điểm khuôn mặt thông qua Face Detection API của Machine Learning để nhận diện khuôn mặt, đem hình ảnh ghi nhận được đem so sánh với hệ thống khuôn mặt các thành viển trong ngôi nhà được lưu trữ trong database của server, nếu nhận diện giống với cái điểm khuôn mặt của một trong những thành viên trong ngôi nhà thì không gửi thông báo qua Telegram chatbot và tiếp tục ghi nhận hình ảnh, nếu server ghi nhận hình ảnh khác với các điểm trên thành viên trong nhà hệ thống sẽ gửi cảnh báo qua Telegram chatbot.

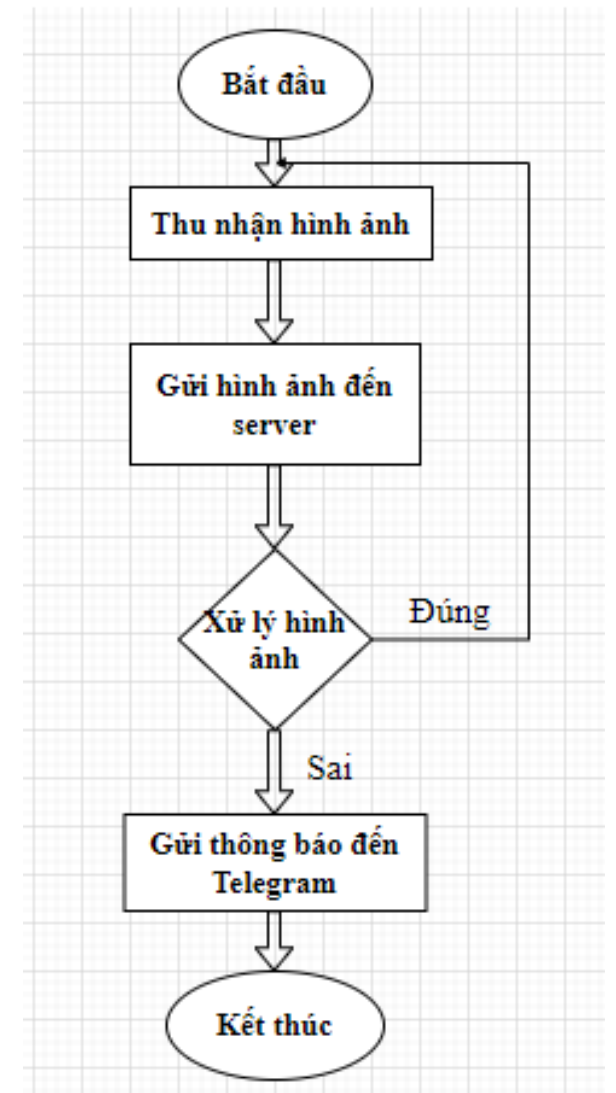

\section{Hình 10. Lưu đồ thuật toán giám sát an ninh trong ngôi nhà}

Các dữ liệu thu thập được thông qua các cảm biến đặt các vị trí phù hợp trong nhà, gửi tín liệu đến ESP8266 NodeMCU xử lý, sử dụng các thư viện phù hợp với các loại cảm biến và các chức năng xây dựng như thư viện DHT xử lý nhiệt độ, độ ẩm; thư viện IRremoteESP8266; ..., tại đây hàm xử lý dữ liệu được xử lý trong hàm "void loop()", xây dựng các kịch bản ở các chế độ tự động ở máy lạnh,kiểm tra các chuyển động của con người, nhận các dữ liệu để xử lý từ người dùng thao tác trên trang web và truyền tín hiệu về thiết bị như máy lạnh, đèn thực hiện cập nhật hiển thị trạng thái như đèn sáng, máy lạnh chuyển nhiệt độ, tăng, giảm nhiệt độ nhận từ ESP8266.

Dữ liệu được xử lý xong gửi đến nơi lưu trữ dữ liệu Firebase Realtime Database thông qua internet, ESP8266 kết nối với Firebase Realtime Database theo tên project đã cài đặt và key do Firebase hỗ trợ để kết nối, câu lệnh kết nối với Firebase Realtime Database: "Firebase.begin (FIREBASE_HOST, FIREBASE_AUTH"

Firebase Hosting cung cấp hosting, xây dựng ứng dụng Web và App với các hosting tĩnh đã được cung cấp sẵn, các kết nối được cung cấp theo tiêu chuẩn bảo mật SSL (Secure Sockets Layer: là tiêu chuẩn của công nghệ bảo mật, truyền thông mã hóa giữa máy chủ Web Server và trình duyệt, tiêu chuẩn này hoạt động và 
đảm bảo dữ liệu truyền tải giữa máy chủ và trình duyệt của người dùng đều riêng tư và toàn vẹn) từ mạng CDN (Content Delivery Network: hệ thống mạng phân tán, mạng lưới máy chủ lưu giữ bản sao của các nội dung tĩnh bên trong website và phân phối đến nhiều máy chủ PoP. Mạng lưới máy chủ CDN được đặt ở khắp mọi nơi trên toàn cầu. Từ $\mathrm{PoP}$ (Points of Presence), dữ liệu sẽ tiếp tục được gửi đến người dùng cuối. Thông qua $\mathrm{CDN}$, bản sao nội dung trên máy chủ gần nhất sẽ được trả về cho người dùng khi họ truy cập website.) [7]

Đoạn mã sau sử dụng $\mathrm{CDN}$ hỗ trợ đưa dữ liệu lên Web và Application, đoạn code là một vài lệnh script về thư viện sử dụng, và đoạn mã code script có chứa key API, đường dẫn Database, ID Project... và câu lệnh dùng để kết nối: "firebase . initiali zeApp (firebaseConfig) ;".

Các dữ liệu ở Firebase Realtime Database được xử lý và hiện thực trên trang web dùng ngôn ngữ Javascript. Xây dựng giao diện Web Application thân thiện, dễ sử dụng được viết bằng CSS, CSS Tailwind.

Trong lưu đồ xử lý chức năng quan sát người lại qua camera trong hệ thống giám sát an ninh của ngôi nhà, camera ghi nhận hình ảnh được kết nối trực tiếp với Server, gửi hình ảnh nhận dạng được về cho Server xử lý. Tại Server ứng dụng thư viện HAAR CASCADE của OpenCV trong lưu trữ dưới dạng Models XML, tại đây sử dụng hai file model phổ biển nhất trong nhận diện khuôn mặt haarcascade_frontalface_default.xml, haarcascade_eye.xml. Lấy các điểm nhận dạng mặt người phổ biến qua các điểm nằm trên vị trí viền dưới khuôn mặt, vị trị tâm mắt trong hai file trên để dạng khuôn mặt người. Thu thập dữ liệu nhận dạng khuôn mặt các thành viên trong gia đình được xây dựng theo ngôn ngữ Python với thư viện OpenCV. Dữ liệu nhận dạng được lưu dưới dạng hình ảnh trong database ở server. Camera ghi nhận hình ảnh xuyên suốt, khi phát hiện khuôn mặt trong bán kính cho phép chuyển hình ảnh đến server, tại đây server xử lý và kiểm tra số điểm nhận diện trên khuôn mặt có trùng khớp với số điểm khuôn mặt các thành viên trong nhà có trong database ở server. Nếu số điểm trên khuôn mặt khớp với dữ liệu có trong database thì sẽ hiển thị qua bên giao diện web tên người được nhận dạng có trong video live từ camera gửi lên web. Nếu số điểm trên khuôn mặt không khớp với dữ liệu thì hệ thống sẽ gửi cảnh báo dưới dạng tin nhắn và hình người lạ đến hộp thư telegram boxchat. Dữ liệu hình ảnh đã được nhận dạng lưu trữ trên Firebase Realtime Database phục vụ phát triển thêm chức năng nhận dạng sau này [8].

\section{KẾT QUẢ TRIỂN KHAI MÔ HÌNH VÀ HƯớNG PHÁT TRIỂN}

\subsection{Kết quả triển khai hệ thống}

Kết quả triển khai hệ thống được thể hiện qua ứng dụng Web Application. Giao diện ứng dụng web, hình 12, thể hiện chức năng xem dự báo thời tiết trong tuần tại các khu vực đang sinh sống. Hiển thị hệ thống các phòng tương ứng với các chức năng trong mỗi phòng, dễ dàng quản lý điều khiển qua lại giữa các phòng.

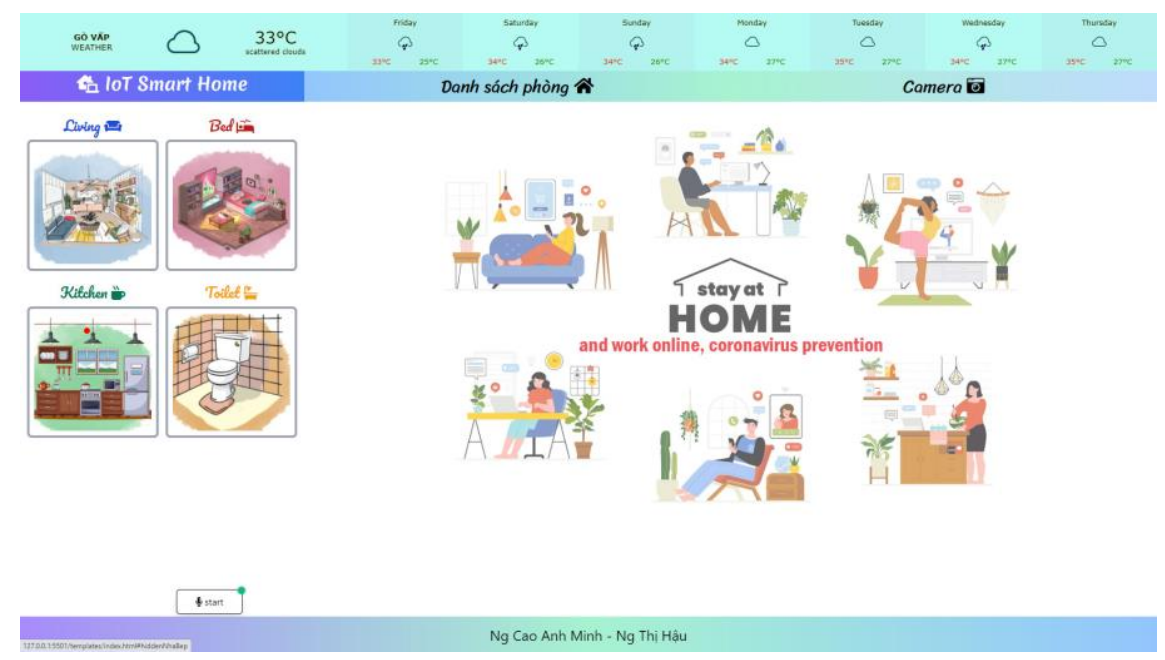

Hình 11. Giao diện chính của trang web

Tại giao diện thể hiện các chức năng điều khiển cho phòng khách, Hình 12, bao gồm: chức năng bật, tắt đèn từ xa - màu vàng của thanh trượt hiển thị đèn đang sáng và màu xám hiển thị đèn đang tắt. Chức năng giám sát nhiệt độ, độ ẩm trong nhà mỗi ngày qua số liệu hiển thị mức cao nhất và thấp nhất trong 24 giờ, 
hiển thị độ ẩm, nhiệt độ 24/24, thống kê số liệu qua biểu đồ line, và xuất file excel ra số liệu nhiệt độ, độ ẩm dễ dàng sử dụng số liệu phát triển thêm chức năng sau này. Chức năng điều khiển máy lạnh với hai chể độ: điều khiển tự động khi người dùng bật chế độ này, hệ thống dựa theo chuyển động của con người để bật, tắt máy lạnh và dựa theo nhiệt độ ngoài trời đo được thông qua API OpenWeather và độ ẩm trong nhà để điều chỉnh nhiệt độ và các chế độ phù hợp; chế độ thứ hai người dùng tự điều khiển qua trang web từ xa điều kiện sử dụng chế độ này khi đã tắt chế độ tự động điều khiển, với chế độ này người dùng tự điều khiển các chế độ và nhiệt độ người dùng mong muốn.

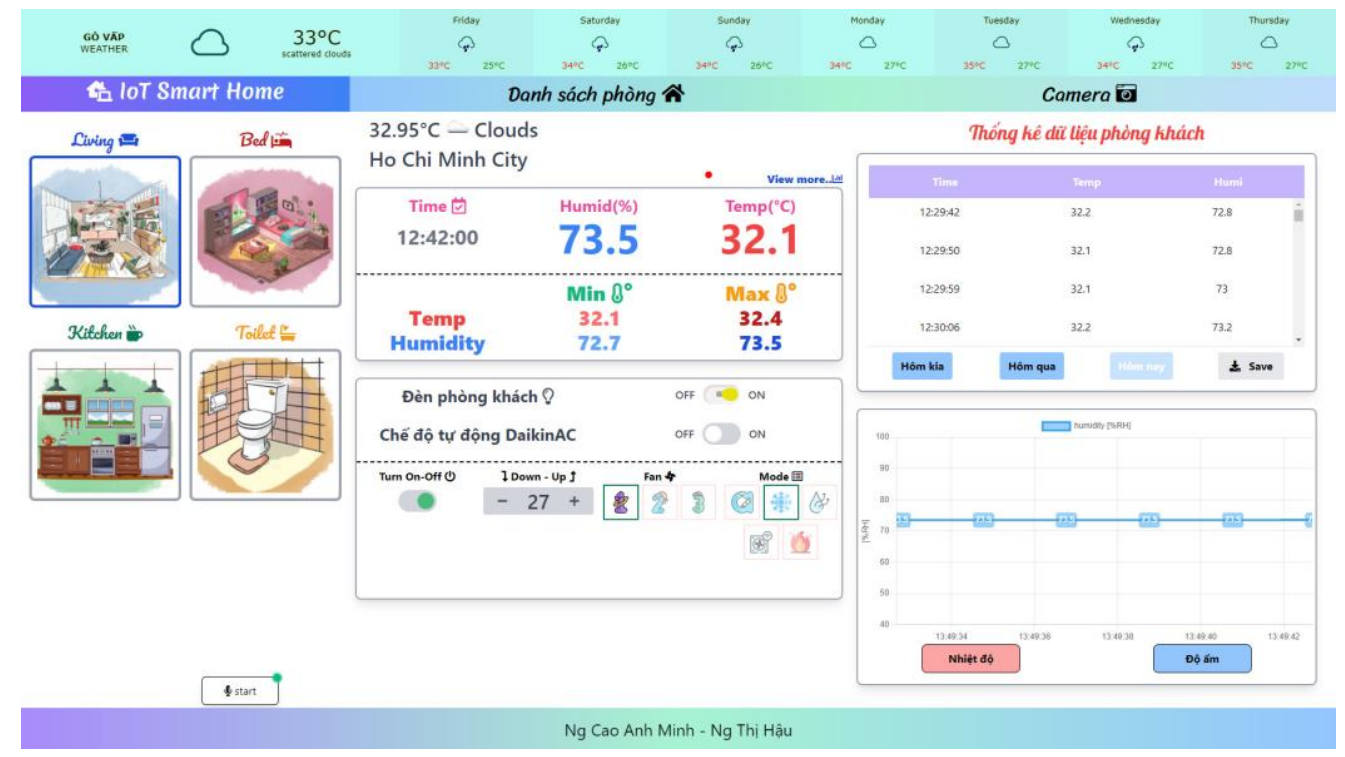

Hình 12. Giao diện thể hiện các chức năng điều khiển thiết bị ở phòng khách

Giao diện điều khiển cho phòng ngủ, Hình 13 , xây dựng thêm chức năng điều khiển mức độ sáng của đèn theo các chế độ sinh hoạt mong muốn như ngủ, học tập với ánh sáng tốt cho mắt,... Xây dựng chức năng điều khiển đèn với hai chế độ: chế độ 1 đèn tự động điều chỉnh mức độ đèn theo ánh sáng trong phòng thông qua cảm biến ánh sáng, đèn phòng ngủ tự điều chỉnh theo kịch bản người dùng đã xây dựng; chế độ thứ 2 người dùng tự điều chỉnh đèn ở theo mức độ mong muốn khi người dùng đã tắt chế độ điều chỉnh đèn tự động.

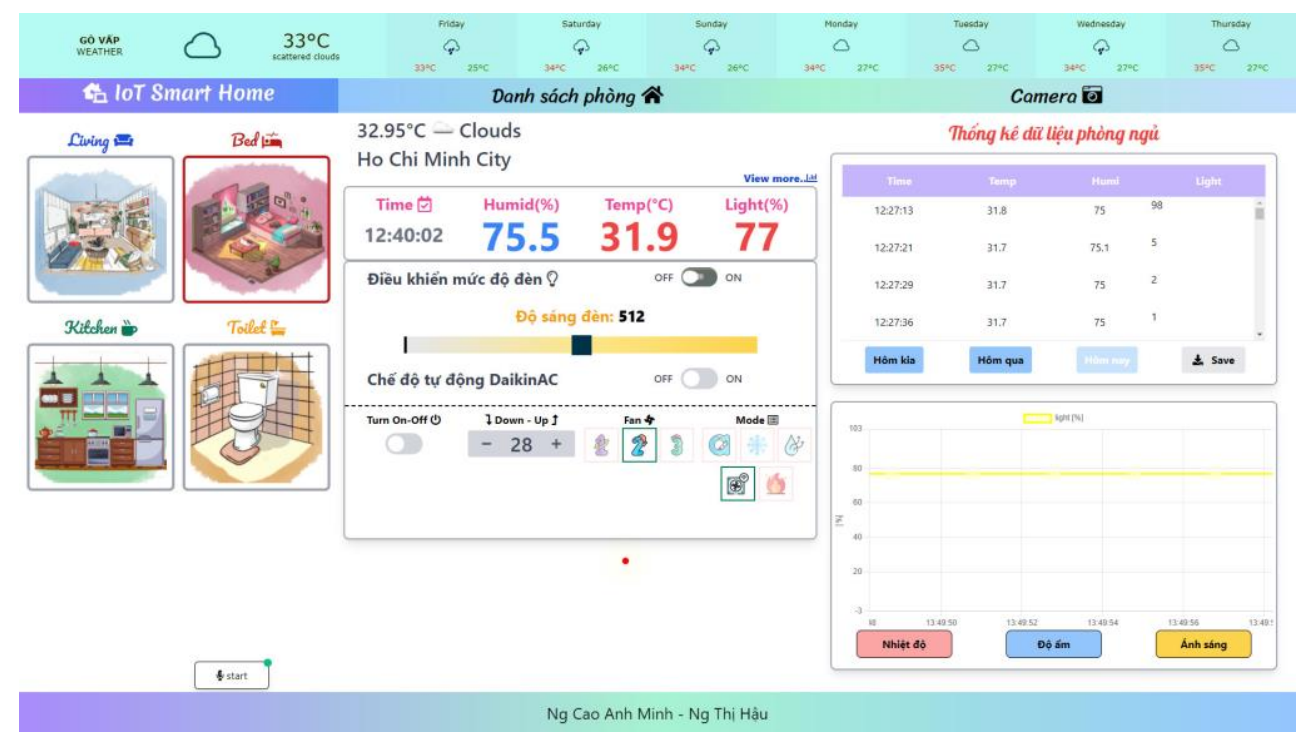

Hình 13. Giao diện thể hiện các chức năng điều khiển thiết bị ở phòng ngủ

Giao diện điều khiển cho nhà bếp, Hình 14, với biểu đồ chứa các mức độ nồng độ gas khi sử dụng với bốn mức độ: Good, Bad, Warning Alarm, Danger Alarm. Mức độ dưới 600 thì hiển thị thông số đo được và 
mức độ cảnh báo là Good, mức độ từ 600 đến dưới 700 hiển thị thông số đo và mức độ cảnh báo là Bad và đồng thời gửi cảnh báo rò rỉ khí gas đến hộp thư telegram boxchat thể hiện ở Hình 15 , mức độ từ hơn 700 đến dưới 800 cảnh báo Warning Alarm, gửi tin nhắn cảnh báo đến hộp thư telegram boxchat và từ hơn 800 cảnh báo Danger Alarm và gửi cảnh báo đến boxchat telegram. Ở chế độ bật, tắt đèn từ xa của nhà bếp tương tự như phòng khách.
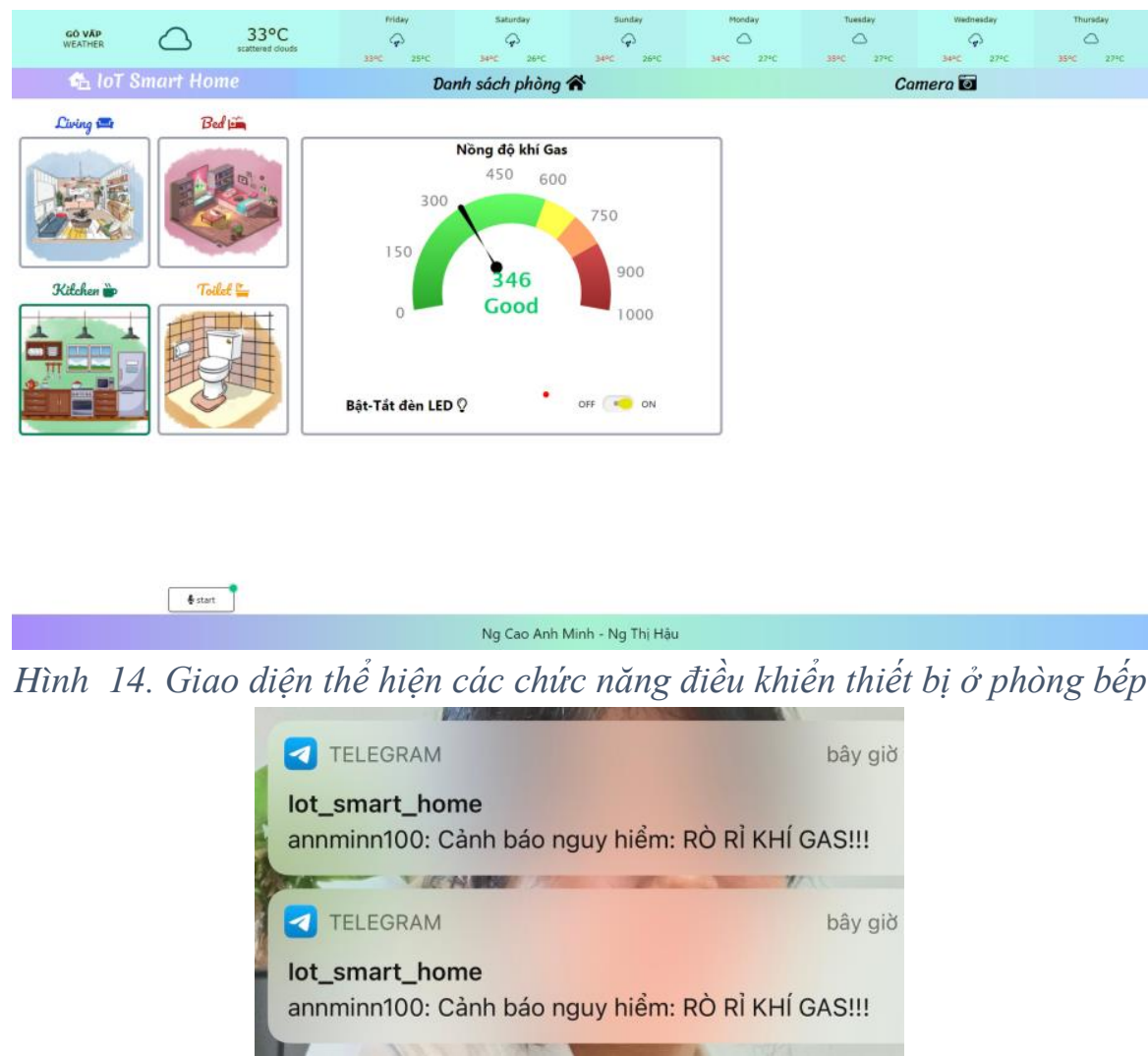

Hình 15. Tin nhắn cảnh báo khí gas vurọt nguõng qua telegram botchat

Giao diện nhà vệ sinh với ánh sáng đèn được bật, tắt theo chuyển động con người thể hiện màu sắc trên trang web màu vàng đèn sáng và màu xám đèn tắt.

Chức năng camera giám sát an ninh được mô phỏng trong Hình 16, hiển thị khuôn mặt thành viên trong gia đình sau khi nhận dạng là thành viên gia đình mà camera đã ghi nhận, hiển thị hình ảnh và tên thành viên. Với các hình ảnh nhận diện người lạ hệ thống gửi cảnh báo hình ảnh và thời gian nhận diện hình ảnh đến hộp thư telegram boxchat như Hình 17.

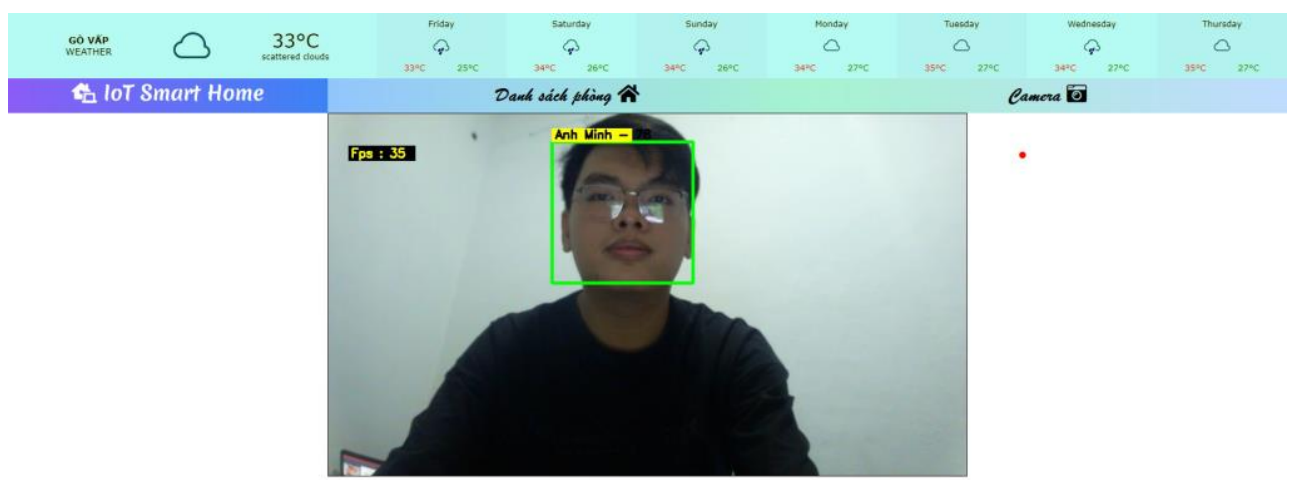

Hình 16. Giao diện nhận dạng thành viên gia đình 


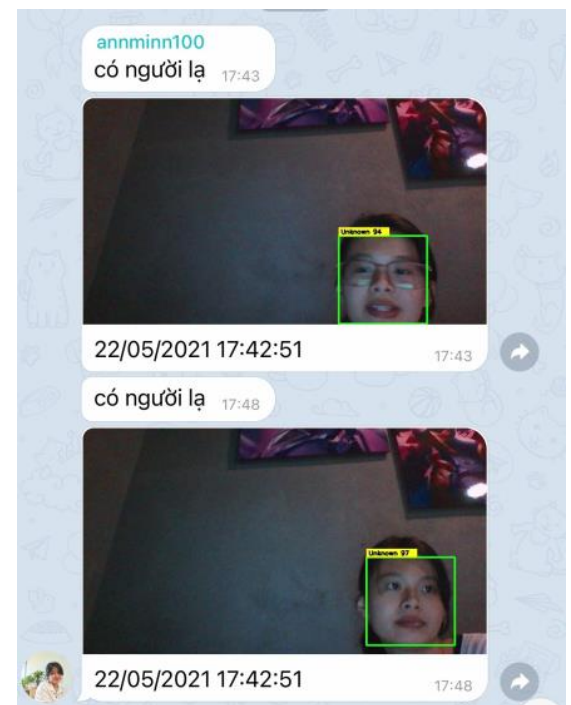

Hình 17. Tin nhắn cảnh báo người ra qua hộp thu telegram boxchat

\subsection{Kết luận và hướng phát triển}

Nhà thông minh đã và đang là xu hướng phát triển trên thế giới khi công nghệ thông tin, đặc biệt là Internet đang áp dụng mọi mặt trong đời sống xã hội ngày nay. Trong khuôn khổ của bài nghiên cứu này, nhóm chúng tôi đã xây dựng thành công hệ thống mô phỏng nhà thông minh sử dụng công nghệ IoT. Hệ thống tích hợp được phần cứng và phần mềm cho chạy trên các máy tính cá nhân, các thiết bị smartphone. Hệ thống ngôi nhà thông minh tự điều chỉnh đèn, điều hòa theo kịch bản mà chủ nhân ngôi nhà đã cài đặt, tích hợp camera an ninh quan sát trong ngôi nhà, hệ thống cảnh báo về rò rỉ khí gas. Tuy nhiên các kịch bản trong ngôi nhà còn hạn chế và các thiết bị, các chức năng chưa được tối ưu hóa một cách hiện đại, phù hợp với thời đại công nghệ và bảo mật tốt nhất.

Hướng phát triển hệ thống nhóm hướng đến là xây dựng hệ thống an ninh bảo mật nhận diện khuôn mặt khi vào nhà, hệ thống điều khiển thiết bị bằng giọng nói, tích hợp các thiết bị tốt cho hệ thống tối ưu hơn.

\section{REFERENCES}

[1] Gaurav Tripathi, Dhananjay Singh, and Antonio J. Jara, “A survey of Internet-of-Things: Future Vision, Architecture, Challenges and Service”, IEEE World Forum on Internet of Things (WF-IoT), 2014, pp. 287 - 292

[2] Vittorio Miori, and Dario Russo, "Domotic evolution towards the IoT", 28th International Conference on Advanced Information Networking and Applications Workshops, 2014, pp. 809-814

[3] Menachem Dom, "Smart Home Systems Based on Internet of Things", Internet of Things (IoT) for Automated and Smart Applications, DOI: 10.5772/intechopen.84894

[4] Min Li, Wenbin Gu, Wei Chen, Yeshen He, Yannian Wu, Yiying Zhang, "Smart Home: Architecture, Technologies and Systems", Procedia Computer Science, Volume 131, 2018, Pages 393-400, ISSN 1877-0509, https://doi.org/10.1016/j.procs.2018.04.219.

[5] https://www.arduino.cc/education

[6] https://firebase.google.com/docs

[7] https://wiki.matbao.net/firebase-la-gi-giai-phap-lap-trinh-khong-can-backend-tu-google/

Ngày nhận bài: 28/04/2021

Ngày chấp nhận đăng:17/07/2021 